\title{
Genome sequence of Vibrio sp. OULL4 isolated from shellfish
}

\author{
Vengadesh Letchumanan ${ }^{1 *}$, Wen-Si Tan ${ }^{2}$, Wai-Fong Yin ${ }^{3}$, Kok-Gan Chan ${ }^{3,4}$ \\ ${ }^{1}$ Novel Bacteria and Drug Discovery Research Group (NBDD), Microbiome and Bioresource Research Strength (MBRS), \\ Jeffrey Cheah School of Medicine and Health Sciences, Monash University Malaysia, 47500 Bandar Sunway, Selangor \\ Darul Ehsan, Malaysia \\ ${ }^{2}$ Illumina Singapore Pte Ltd, Woodlands Industrial Park E1, Singapore \\ ${ }^{3}$ Division of Genetics and Molecular Biology, Institute of Biological Sciences, Faculty of Science, University of Malaya, \\ 50603 Kuala Lumpur, Malaysia \\ ${ }^{4}$ International Genome Centre, Jiangsu University, Zhenjiang 212013, PR China
}

\begin{abstract}
The members of Vibrionaceae family are Gram-negative bacterium are ubiquitous in marine and estuarine environments. This diverse group of bacteria include many pathogenic strains that potentially cause infection to human and aquaculture animals. Vibrio cholerae, Vibrio parahaemolyticus and Vibrio vulnificus are among the few recognized as a major, worldwide cause gastroenteritis, particularly in countries where seafood consumption is high. The control of these vibrios has been a hurdle due to the rising numbers of antibiotic resistant strains in the environments. We report the genome sequence of Vibrio sp. OULL4 isolated from shellfish. The availability of this genome sequence will facilitate the study of its antimicrobial traits, as well as add our knowledge of Vibrio sp. diversity and evolution.
\end{abstract}

Keywords: Vibrionaceae; infection; gastroenteritis; antibiotic; genome

Received: $27^{\text {th }}$ February 2020

Accepted: $24^{\text {th }}$ March 2020

Published Online: $6^{\text {th }}$ April 2020

*Correspondence: Vengadesh Letchumanan, Jeffrey Cheah School of Medicine and Health Sciences, Monash University Malaysia; vengadesh.letchumanan1@monash.edu.

Citation: Letchumanan V, Tan W-S, Yin W-F et al. Genome sequence of Vibrio sp. OULL4 isolated from shellfish. Prog Microbe Mol Biol 2020; 3(1): a0000066. https://doi.org/10.3687/pmmb.a0000066

\section{Introduction}

Seafood production has double over the years to meet the rising consumer demand for seafood. This involuntary action has exposed aquatic animals to bacterial infections ${ }^{[1,2]}$. This situation gets complicated and worsen by the emergence of resistant Vibrio sp. strains, which hampers medical care. Vibrio sp. is a Gram-negative halophilic bacteria that belongs to the Vibrionaceae family ${ }^{[3-8]}$. They naturally inhabit the aquatic surroundings and associated with aquatic animals for example crustaceans, molluscs and fish ${ }^{[9-13]}$.

The World Health Organization (WHO) has acknowledged antibiotic resistance as a public health hazard that affects millions of people worldwide ${ }^{[14]}$. Due to excessive use of antibiotics in the aquaculture sector, the incidence of resistance accelerated, mostly among foodborne pathogens such as Vibrio sp. ${ }^{[15-23]}$, Listeria sp. ${ }^{[24-26]}$, and Salmonella $\mathrm{sp}^{[27-32]}$. The resistant foodborne pathogens poses a threat and challenge to drug discovery programmes worldwide ${ }^{[33,34]}$. Therefore, it is important to continuously monitor and manage the resistant Vibrio sp. in seafood and environments.
Vibrio sp. OULL4 strain was isolated from shellfish originated from a supermarket in Selangor, Malaysia. The strain presented a large yellow colony on selective media - thiosulphate citrate bile salt sucrose (TCBS) agar. The antibiotic susceptibility test was performed to determine to resistance phenotype of Vibrio sp. OULL4 strain. The strain was resistant to $11 / 14$ antibiotics tested, namely the ampicillin, ampicillin/sulbactam, $3^{\text {rd }}$ generation cephalosporin (cefotaxime, ceftazidime), aminoglycoside (amikacin, gentamicin, kanamycin), suphamethox/trimethoprim, oxytetracycline, tetracycline, and chloramphenicol. This is a worrying scenario as the antibiotic resistant profile exhibited by the strain is among the recommended antibiotics agents used in treatment if Vibrio sp. infection ${ }^{[35-37]}$. The Vibrio sp. OULL4 strain was selected for genome sequencing to further explore and understand the antibiotic resistant traits.

\section{Data description}

The genomic DNA of Vibrio sp. OULL4 was extracted using Masterpure ${ }^{\mathrm{TM}}$ DNA purification kit (Epicentre, Il lumina Inc., Madison, WI, USA) prior to RNase (Qiagen, 
USA) treatment ${ }^{[38,39]}$. The DNA quality was quantified using NanoDrop spectrophotometer (Thermo Scientific, Waltham, MA, USA) and a Qubit version 2.0 fluorometer (Life Technologies, Carlsbad, CA, USA). Illumina sequencing library of genomic DNA was prepared using Nextera ${ }^{\mathrm{TM}}$ DNA Sample Preparation kit (Illumina, San Diego, CA, USA) and library quality was validated by a Bioanalyzer 2100 high sensitivity DNA kit (Agilent Technologies, Palo Alto, CA) prior to sequencing. The genome of OULL4 strain was sequenced on MiSeq platform with MiSeq Reagent Kit 2 (2 x 250bp; Illumina Inc, San Diego, CA, USA) $)^{[40]}$. The trimmed sequences were de novo assembled with CLC Genomic Workbench version 5.1 (CLC Bio, Denmark). Contigs with at least $200 \mathrm{bp}$ and 30-fold coverage were selected for gene prediction and annotation. The bacteria identity was also checked by local BLAST against NCBI prokaryotic 16S rRNA database. Prodigal (version 2.6.1) was utilized to predict the bacteria gene coding sequence (CDS) from the draft genome ${ }^{[41]}$. Gene annotation was performed by local BLAST of translated predicted CDS against NCBI-nr database and on Rapid Annotation using Subsystem Technology (RAST) server ${ }^{[42]}$. Presence of rRNA and tRNA genes were detected using RNAmmer and tRNAscan SE version $1.21^{[43,44]}$ A total of 59 contigs were generated with N50 size of 201,133bp. The assembled genome size of Vibrio sp. OULL4 contains 4,146,642 bp, with an average genome coverage of 54 -fold with a $\mathrm{G}+$
C content of $45.4 \%$ (Table 1). The whole genome project was deposited at DDBJ/EMBL/GenBank under accession MQVK00000000. The version described in this paper is the first version MQVJ00000000. It is composed of 59 contigs and there were 3,743 protein coding genes (out of a total of 3,898 predicted gene) (Table 1).

Table 1. General features of Vibrio sp. OULL4 draft genome.

\begin{tabular}{ll}
\hline Attribute & Value \\
\hline Genome size (bp) & $4,146,642$ \\
G + C content \% & 45.4 \\
DNA scaffold & 59 \\
Total genes & 3,898 \\
Protein coding genes & 3,743 \\
RNA genes (5S, 16S, 24S) & $5,3,1$ \\
Pseudo genes & 55 \\
\hline
\end{tabular}

The analysis obtained from RAST server revealed 493 subsystems (Figure 1). The annotated genome has 63 genes responsible for resistance to antibiotic and toxic compounds including 25 genes for multidrug resistance efflux pumps, one gene for beta-lactamase, and two genes for tetracycline resistance. The presences of these genes in the genome is closely related to the phenotypic resistance exhibited by the strain toward ampicillin, cefotaxime, oxytetracycline, and tetracycline.

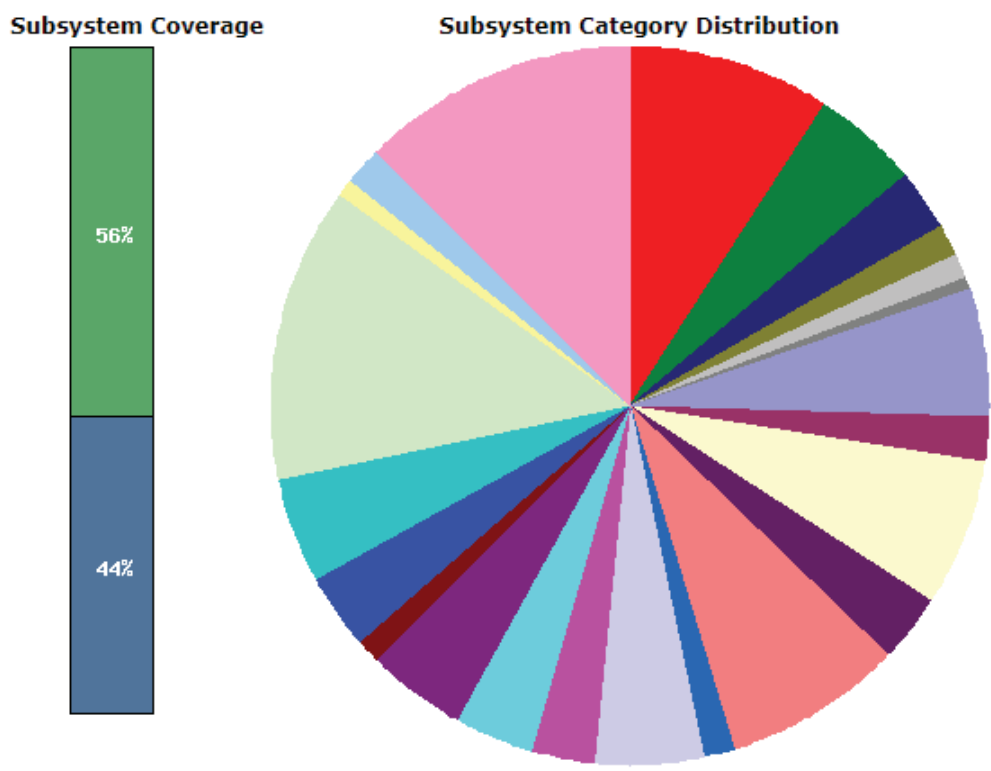

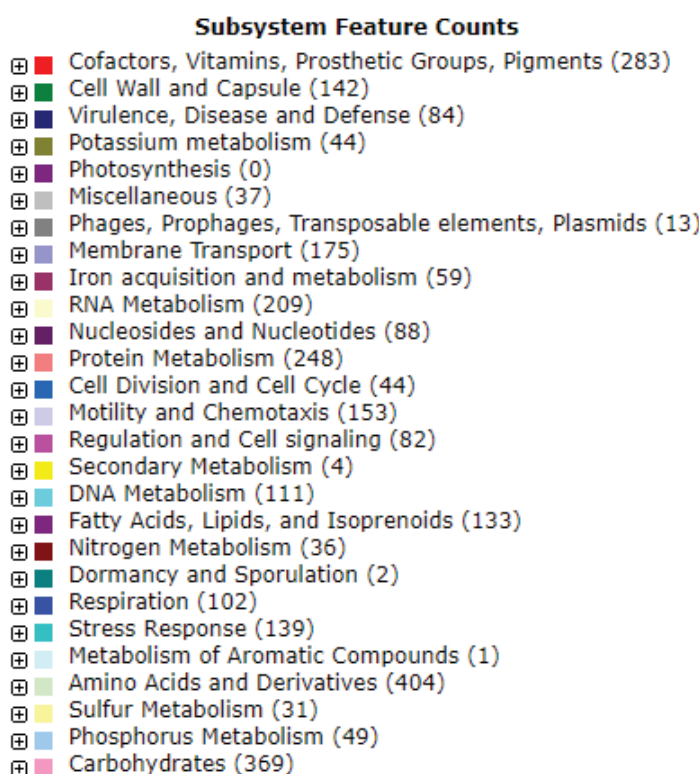

Figure 1. Subsystem category distribution of Vibrio sp. OULL4 (based on RAST annotation server)

Vibrio sp. OULL4 is a multidrug resistant strain-resistant to $11 / 14$ antibiotics tested. The resistant phenotype and genes of genome illustrates how extensive antibiotics have been used in aquaculture sector. Some of the resistance phenotype seen in this strain possibly due to the misuse of permitted antibiotics in Asian aquaculture industry namely tetracycline, quinolone, oxytetracycline, sulphonamide, and trimethoprim ${ }^{[45]}$. Soon, our dependency to antibiotics will eventually deprive the efficacy of clinical antibiotics. We will need to resort to non-antibiotic approach such as bacteriophage application or natural plant antimicrobials to manage Vibrio infections in the aquaculture ${ }^{[46-50]}$. We also could adapt quorum sensing method to understand the various signalling molecules of Vibrio sp. These information are useful in the management of virulence traits ${ }^{[51]}$. In summary, the application of antibiotics in aquaculture should be reviewed and monitored in order to ensure the efficacy of these antibiotics for treatment.

\section{Conflict of Interest}

The authors declare that the research was conducted in 
the absence of any commercial or financial relationships that could be construed as a potential conflict of interest.

\section{Acknowledgement}

This work was supported by University of Malaya for High Impact Research Grant (UM-MOHE HIR Nature Microbiome Grant No. H-50001-A000027 and No. A000001-50001) and PPP Grant (PG090-2015B) awarded to K-GC.

\section{Authors Contributions}

The research and manuscript writing were performed by VL and W-ST. W-FY and K-GC provided vital guidance and support for the success of the project. The project was founded by VL and K-GC.

\section{Reference}

1. Tan LT-H, Lee L-H, Goh B-H. The bioprospecting of anti-Vibrio Streptomyces species: Prevalence and applications. Prog Microb Mol Biol 2019; 1(1).

2. Hamdan RH, Peng TL, Ong B, et al. Antibiotics resistance of Vibrio spp. isolated from diseased seabass and tilapia in cage culture. 2018.

3. Letchumanan V, Chan K-G, Lee L-H. Vibrio parahaemolyticus: A review on the pathogenesis, prevalence, and advance molecular identification techniques. Front Microbiol 2014; 5: 705.

4. Letchumanan V, Chan K-G, Lee L-H. An insight of traditional plasmid curing in Vibrio species. Front Microbiol 2015; 6: 735.

5. Sawabe T, Ogura Y, Matsumura Y, et al. Updating the Vibrio clades defined by multilocus sequence phylogeny: Proposal of eight new clades, and the description of Vibrio tritonius sp. nov. Front Microbiol 2013; 4: 414

6. Lee L-H and Raghunath P. Vibrionaceae diversity, multidrug resistance and management. Front Microbiol 2018; 9: 563.

7. Baker-Austin C, Oliver JD, Alam M, et al. Vibrio spp. infections. Nat Rev Dis Primers 2018; 4(1): 1-19.

8. Ceccarelli D, Amaro C, Romalde JL, et al. Vibrio Species. Food Microbiology: Fundamentals and frontiers. 2019: 347-88.

9. Elmahdi S, DaSilva LV, Parveen S. Antibiotic resistance of Vibrio parahaemolyticus and Vibrio vulnificus in various countries: A review. Food Microbiol 2016; 57: 128-34

10. Roux FL, Wegner KM, Baker-Austin C, et al. The emergence of Vibrio pathogens in Europe: Ecology, evolution, and pathogenesis (Paris, 11-12th March 2015). Front Microbiol 2015; 6: 830.

11. Robert-Pillot A, Copin S, Himber C, et al. Occurrence of the three major Vibrio species pathogenic for human in seafood products consumed in France using real-time PCR. Int J Food Microbiol 2014; 189: 75-81.

12. Bonnin-Jusserand M, Copin S, Le Bris C, et al. Vibrio species involved in seafood-borne outbreaks (Vibrio cholerae, V. parahaemolyticus and $V$. vulnificus): Review of microbiological versus recent molecular detection methods in seafood products. Crit Rev Food Sci Nutr. 2019;59(4):597-610.

13. Raghunath P. Roles of thermostable direct hemolysin (TDH) and TDHrelated hemolysin (TRH) in Vibrio parahaemolyticus. Front Microbiol. 2015;5:805.

14. Aslam B, Wang W, Arshad MI, et al. Antibiotic resistance: a rundown of a global crisis. Infect Drug Resist. 2018;11:1645.

15. Letchumanan V, Yin W-F, Lee L-H, et al. Prevalence and antimicrobial susceptibility of Vibrio parahaemolyticus isolated from retail shrimps in Malaysia. Front Microbiol. 2015;6:33.

16. Letchumanan V, Pusparajah P, Tan LT-H, et al. Occurrence and antibiotic resistance of Vibrio parahaemolyticus from shellfish in Selangor, Malaysia. Front Microbiol. 2015;6:1417.

17. Letchumanan V, Chan K-G, Khan TM, et al. Bile sensing: The activation of Vibrio parahaemolyticus virulence. Front Microbiol. 2017;8:728.

18. Lee L-H, Ab Mutalib N-S, Law JW-F, et al. Discovery on antibiotic resistance patterns of Vibrio parahaemolyticus in Selangor reveals carbapenemase producing Vibrio parahaemolyticus in marine and freshwater fish. Front Microbiol. 2018;9:2513.

19. Heng S-P, Letchumanan V, Deng C-Y, et al. Vibrio vulnificus: an environmental and clinical burden. Front Microbiol. 2017;8:997.

20. Law JWF, Letchumanan V, Chan KG, et al. Insights into detection and identification of foodborne pathogens. Food Borne Pathogens and Antibiotic Resistance. 2016
21. Letchumanan V, Ab Mutalib N-S, Wong SH, et al. Determination of antibiotic resistance patterns of Vibrio parahaemolyticus from shrimp and shellfish in Selangor, Malaysia. Prog Microb Mol Biol. 2019;1(1).

22. Letchumanan V, Loo K-Y, Law JW-F, et al. Vibrio parahaemolyticus: The protagonist of foodborne diseases. Prog Microb Mol Biol. 2019;1(1).

23. Zavala-Norzagaray AA, Aguirre AA, Velazquez-Roman J, et al. Isolation, characterization, and antibiotic resistance of Vibrio spp. in sea turtles from Northwestern Mexico. Front Microbiol. 2015;6:635.

24. Letchumanan V, Wong P-C, Goh B-H, et al. A review on the characteristics, taxanomy and prevalence of Listeria monocytogenes. Prog Microb Mol Biol. 2018;1(1)

25. Law JW-F, Ab Mutalib N-S, Chan K-G, et al. An insight into the isolation, enumeration, and molecular detection of Listeria monocytogenes in food. Front Microbiol. 2015;6:1227

26. Olaimat AN, Al-Holy MA, Shahbaz HM, et al. Emergence of antibiotic resistance in Listeria monocytogenes isolated from food products: a comprehensive review. Compre Rev Food Sci Food Safety. 2018;17(5):1277-92.

27. Eng S-K, Pusparajah P, Ab Mutalib N-S, et al. Salmonella: a review on pathogenesis, epidemiology and antibiotic resistance. Frontiers in Life Science. 2015;8(3):284-93.

28. Khoo C-H, Cheah Y-K, Lee L-H, et al. Virulotyping of Salmonella enterica subsp. enterica isolated from indigenous vegetables and poultry meat in Malaysia using multiplex-PCR. Antonie Van Leeuwenhoek. 2009;96(4):441.

29. Yoke Kqueen C, Learn Han L, Noorzaleha A, et al. Characterization of multiple antimicrobial resistant Salmonella enterica subsp. enterica isolated from indigenous vegetables and poultry in Malaysia. Lett Appl Microbiol. 2008;46(3):318-24.

30. Learn-Han L, Yoke-Kqueen C, Salleh NA, et al. Analysis of Salmonella Agona and Salmonella Weltevreden in Malaysia by PCR fingerprinting and antibiotic resistance profiling. Antonie Van Leeuwenhoek. 2008;94(3):377.

31. Learn-Han L, Yoke-Kqueen C, Shiran M, et al. Molecular characterization and antimicrobial resistance profiling of Salmonella enterica subsp. enterica isolated from 'Selom'(Oenanthe stolonifera). Inter Food Res J. 2009;16(1):191-202.

32. Cheah Y-K, Salleh NA, Lee L-H, et al. Comparison of PCR fingerprinting techniques for the discrimination of Salmonella enterica subsp. enterica serovar Weltevreden isolated from indigenous vegetables in Malaysia. World J Microbiol Biotech. 2008;24(3):327.

33. Alanis AJ. Resistance to antibiotics: are we in the post-antibiotic era? Arch Med Res. 2005;36(6):697-705.

34. Morehead MSandScarbrough C. Emergence of global antibiotic resistance. Prim Care. 2018;45(3):467-84.

35. Letchumanan V, Ser H-L, Chan K-G, et al. Genome sequence of Vibrio parahaemolyticus VP103 strain isolated from shrimp in Malaysia. Front Microbiol. 2016;7:1496.

36. Letchumanan V, Ser H-L, Tan W-S, et al. Genome sequence of Vibrio parahaemolyticus VP152 strain isolated from Penaeus indicus in Malaysia. Front Microbiol. 2016; 7:1410.

37. Letchumanan V, Ser H-L, Tan W-S, et al. Genome sequence of Vibrio sp. SALL 6 isolated from shellfish. Prog Microb Mol Biol. 2019;2(1).

38. Ser H-L, Tan W-S, Ab Mutalib N-S, et al. Draft genome sequence of mangrove-derived Streptomyces sp. MUSC 125 with antioxidant potential. Front Microbiol. 2016;7:1470.

39. Ser H-L, Tan W-S, Ab Mutalib N-S, et al. Genome sequence of Streptomyces mangrovisoli MUSC 149T isolated from intertidal sediments. Braz J Microbiol. 2018;49(1):13-5.

40. Kher H-L, Krishnan T, Letchumanan V, et al. Characterization of quorum sensing genes and $\mathrm{N}$-acyl homoserine lactones in Citrobacter amalonaticus strain YG6. Gene. 2019;684:58-69.

41. Hyatt D, Chen G-L, LoCascio PF, et al. Prodigal: prokaryotic gene recognition and translation initiation site identification. BMC Bioinformatics. 2010;11(1):119.

42. Aziz RK, Bartels D, Best AA, et al. The RAST Server: rapid annotations using subsystems technology. BMC Genomics. 2008;9(1):75.

43. Lowe T and Eddy SR. tRNAscan-SE: a program for improved detection of transfer RNA genes in genomic sequence. Nucleic Acids Res. 1997;25(5):955-64.

44. Lagesen K, Hallin P, Rødland EA, et al. RNAmmer: consistent and rapid annotation of ribosomal RNA genes. Nucleic Acids Res. 2007;35(9):31008.

45. Yano Y, Hamano K, Satomi M, et al. Prevalence and antimicrobial susceptibility of Vibrio species related to food safety isolated from shrimp cultured at inland ponds in Thailand. Food Cont. 2014;38:30-6.

46. Letchumanan V, Chan K-G, Pusparajah P, et al. Insights into bacteriophage application in controlling Vibrio species. Front Microbiol. 2016;7:1114

47. Ma DS, Tan LT-H, Chan K-G, et al. Resveratrol-potential antibacterial agent against foodborne pathogens. Front Pharmacol. 2018;9:102.

48. Tan T, Chan K, Lee L. Application of bacteriophage in biocontrol of major foodborne bacterial pathogens. J Mol Biol Mol Imaging. 2014;1(9).

49. Yen M, Cairns LS, Camilli A. A cocktail of three virulent bacteriophages prevents Vibrio cholerae infection in animal models. Nat Commun. 2017;8(1):1-7.

50. Quiroz-Guzmán E, Peña-Rodriguez A, Vázquez-Juárez R, et al. Bacteriophage cocktails as an environmentally-friendly approach to prevent Vibrio parahaemolyticus and Vibrio harveyi infections in brine shrimp (Artemia franciscana) production. Aquac. 2018;492:273-9.

51. Tan W-S, Law JW-F, Letchumanan V, et al. Decoding the mystery of how bacteria "talk": Among Gram-negative microorganisms. Prog Microb Mol Biol. 2019;2(1). 